\title{
MedienPädagogik
}

Zeitschrift für Theorie und Praxis der Medienbildung

Themenheft Nr. 30: Medienpädagogik und Erwachsenenbildung. Herausgegeben von Bernhard Schmidt-Hertha und Matthias Rohs.

\section{Leitideen in der medienpädagogischen Erwachsenenbildung: Ein analytischer Blick}

Christian Helbig und Sandra Hofhues

\begin{abstract}
Zusammenfassung
Der Beitrag geht den Fragen nach, welche Leitideen des medienpädagogischen Diskurses für die Erwachsenenbildung besonders relevant sind. Umgekehrt wird gefragt, welche medienpädagogischen Leitideen sich in der erwachsenenbildnerischen Praxis (bereits) wiederfinden. Auf diese Weise werden Synergien zwischen Medienpädagogik und Erwachsenenbildung offengelegt, um dafür zu sensibilisieren, dass konkrete Medien-Phänomene aufgrund ihrer Vielfalt und bisweilen Ambivalenzen reflektierte pädagogische Forschung und Praxis im Austausch zwischen den pädagogischen Teildisziplinen erfordern.
\end{abstract}

Guidelines and practices of media pedagogy in adult education. An analytic view

\begin{abstract}
This paper examines questions about the relevance of guiding principles at the discourse of media pedagogy for adult education. Conversely, it is asked, which guiding principles at the basis of media pedagogy can be found in the practice of adult education. That way, we will disclose synergies between media pedagogy and adult education, to sensitise for a reflective research and practice related to a debate between the pedagogical disciplines about specific media-phenomena because of their variety and occasional ambivalence.
\end{abstract}

Medien in der Erwachsenbildung: Sondierung eines Feldes aus medienpädagogischer Sicht

Angesichts der Diskussionen um selbstgesteuertes und selbstorganisiertes Lernen in Unternehmen spielen seit den 1990er Jahren u.a. computerbasierte, später webbasierte und heute gamifizierte Lern-Formate eine wichtige Rolle. Sie werden umgesetzt in Form von internen Computer-based Trainings (CBTs)/Web-based Trainings (WBTs) oder E-Learning/Blended Learning im geschützten (semi-)virtuellen Raum. Dabei zeigt sich, dass vielfach auf Begriffe, Konzepte und Ansätze aus der Medienpädagogik Bezug genommen wurde, insbesondere in der beruflichen (Erwachsenen-) Bildung aber ein Schwerpunkt auf die Förderung technisch-funktionaler Medienkompetenzen und auf die Mediendidaktik gelegt wurde. Damit wurde Medienpädagogik 
verengt auf die Aneignung bestimmter Medien für das Lernen und die Organisation von mediengestütztem Lernen innerhalb eines formal geplanten Zusammenhangs.

In dieser Perspektive der beruflichen (Erwachsenen-)Bildung mit mediendidaktischen (insbesondere bildungstechnologischen) Schwerpunkten sind die generellen Anforderungen an Medienkompetenz-Entwicklung und Strukturen für Medienbildung im Erwachsenenalter in den Hintergrund gerückt. Grell (2017, 80 ff.) gibt in ihrem Beitrag zur Erwachsenbildung erst kürzlich Hinweise darauf, dass sich die Diskussion um Medien in der Erwachsenenbildung grundlegend auf zwei Bereiche eingrenzen lässt. Erstens der Wandel der Lernkultur durch digitale Technologien zwischen Subjekt, Medien und der formalen (Organisation von) Bildung. Hier kommt die Sicht der Mediendidaktik auf das formal organisierte Lernen mit Medien besonders zum Tragen. Zweitens besteht die subjektive Anforderung, ständig über bzw. durch Medien neues Wissen zu erwerben, neue Fähigkeiten und neue Handlungsroutinen zu entwickeln und veraltete Handlungsmuster abzulegen. In dieser Sichtweise rückt das Subjekt und die Relevanz von Medienhandeln in unterschiedlichen Kontexten in den Mittelpunkt. Medienhandeln im Beruf ist häufig einer davon.

Folglich erweist sich das Feld der Medien in der Erwachsenenbildung als besonders geeignet, jenseits aktueller, öffentlicher Debatten um «Digitalisierung», «digitale Transformation» oder "Computerisierung von Arbeit» sowie «Industrie 4.0»(vgl. Scharnberg et al. 2017; Olbermann 2016; Seifert 2016; Herber et al. 2013) den Brückenschlag zwischen Medienpädagogik und Erwachsenenbildung zu gestalten. Schliesslich stellte Hüther bereits 1994 fest, dass es

«[n]icht selten [...] dieselben Umbruchsituationen [sind], die Erwachsenenbildung und Medienpädagogik gleichermassen zwingen und sie in Kooperation bringen, indem entweder Erwachsenenbildung vorhandene medienpädagogische Konzeptionen aufgreift [...] oder indem sich Medienpädagogik in Problem- und Bedarfslagen der Erwachsenenbildung einbringt.» (Hüther 1994, 290)

Nähert man sich dem Feld der Medien in der Erwachsenenbildung, erfordert jedoch die heterogene Zielgruppe, Praktiken der Medienaneignung in diversen Altersgruppen über das Narrativ der Digital Natives (Hepp et al. 2014, 22) hinaus einzubeziehen und nach Konzepten für diverse Bildungsbiografien zu suchen. Aufgrund unserer eigenen Verortung in der Medienpädagogik stellt sich nicht zuletzt die Frage danach, wie es gelingen kann, in der Erwachsenenbildung eine medienpädagogische Perspektive zu stärken, die über mediendidaktische Handlungsweisen hinausgeht. Entsprechend leiten uns folgende beiden Fragestellungen durch den gesamten Beitrag:

1. Welche Leitideen des medienpädagogischen Diskurses wären für die Erwachsenenbildung besonders relevant?

2. Welche medienpädagogischen Leitideen finden sich bereits in der erwachsenenbildnerischen Praxis wieder? 
Auf diese Weise möchten wir Synergien zwischen den Diskursen offenlegen, um dafür zu sensibilisieren, dass konkrete Medien-Phänomene aufgrund ihrer Vielfalt und bisweilen Ambivalenzen reflektierte pädagogische Forschung und Praxis im Austausch zwischen den pädagogischen Teildisziplinen erfordern.

\section{Leitideen und bedeutende Ansätze der Medienpädagogik: Einladungen an die Er- wachsenenbildung}

Die Medienpädagogik umfasst alle Theorien, Modelle und Ansätze wie auch Konzepte und empirische Studien, die sich mit der Rolle der Medien in Erziehung, formaler, non-formaler und informeller Bildung und Sozialisation befassen (vgl. z.B. Süss et al. 2010, 14). Common Sense ist, dass die Veränderungen und Entwicklungen von Kommunikation und Kommunikationsanforderungen, die heute unter dem Begriff Mediatisierung diskutiert und erforscht werden (vgl. Krotz 2007; 2012), Gesellschaft, Kultur wie auch die Arbeitswelt sowie die politischen und pädagogischen Diskurse nachhaltig prägen. Ebenso wie die Erwachsenenbildung hat die Medienpädagogik ihre pädagogischen Wurzeln in der Aufklärung. Historisch haben sich dann in der pädagogischen Auseinandersetzung mit Medien einige Leitideen herausgebildet, die nach Stölting $(1999,113)$ «legitimierende Idealisierungen» sind. Handlungs- und diskursleitende Prämissen der Medienpädagogik sind dabei 1) die starke Subjektorientierung, 2) die übergeordneten pädagogischen Ziele der Mündigkeit und gesellschaftlichen Teilhabe, 3) die Problematisierung digitaler Ungleichheiten - von Digital Divide bis Alphabetisierung u.a. von Erwachsenen, 4) die (zunehmende) Förderung von Medienkompetenzen und Medienbildungsprozessen in formalen, non-formalen und informellen (Lern-) und (Bildungs-)Kontexten angesichts verschwimmender Generationenkonzepte und Zielgruppen, 5) das unausgesprochene Primat der Handlungsorientierung und 6) ein umfangreicher und nicht bloss technischer Medienbegriff, welcher auch für die Erwachsenbildung zentral sein/werden könnte.

\section{Subjektorientierung}

In der Medienpädagogik besteht weitestgehend Konsens darüber, dass innerhalb der pädagogischen Teildisziplin das Wechselverhältnis von Gesellschaft, Medien und Subjekt verhandelt wird. So steht der Mensch mit ihren/seinen subjektiven Sinnkonstruktionen und ihrem/seinem Handeln im Vordergrund. Gefragt wird u.a., wie sich Subjekte sowohl bestimmte Medienangebote als auch ihre subjektive Umwelt bzw. Kontexte aneignen (vgl. hierzu Vollbrecht/Wegener 2010). Dabei interessiert nicht nur der Aneignungsprozess im technisch-funktionalen Sinne, wie er bspw. bei Tully (2004) beschrieben wird, sondern gerade der kommunikative Aushandlungsprozess 
in der Aneignung zwischen Mensch und Welt (vgl. Krotz 2007; 2012). Für medienpädagogisches Handeln ist folglich das Schaffen von Kommunikationsanlässen mit, über und durch Medien zentral.

\section{Mündigkeit und gesellschaftliche Teilhabe}

Mit Blick auf Forderungen der Aufklärung wird Partizipation als ein fundamentales Recht aller Mitglieder einer Gesellschaft in allen sie betreffenden gesellschaftlichen Bereichen definiert (vgl. Knauer/Sturzenhecker 2005; Stange 2007). Dieser weit gefasste Begriff von Partizipation ist für den Diskurs und die Konzepte der Medienpädagogik deshalb von grosser Bedeutung, da er sich nicht nur auf den Arbeitsmarkt oder die Beschäftigungsfähigkeit bezieht, sondern den politischen, sozialen und kulturellen Bereich einbezieht. Tulodziecki arbeitet bspw. heraus, dass «der mündige Umgang mit Medien zur Förderung von Bildung und Demokratie, Wirtschaft und Kultur eine [...] Leitidee der Medienpädagogik dar[stellt], die heute zum Teil im Begriff der Medienkompetenz dominant ist» (Tulodziecki 1998, 13). Entsprechend divers sind medienpädagogische Konzepte: Sie reichen von Kommunikationsanlässen bis hin zu Maker Spaces, in denen Subjekte Medien «zum Be-Greifen wesentlicher Konzepte der Gegenwart» (Schelhowe 2008) nutzen und mit denen sie Gesellschaft mündig mit-gestalten sollen (vgl. Paus-Haase et al. 2002, 11f.). Insbesondere die Mitglieder der «Klagenfurter Projektgruppe Medienerziehung» führen das Leitziel der «Medienmündigkeit» in die Medienpädagogik ein und begreifen diese als Übernahme von Selbstbestimmung und sozialer Verantwortung (vgl. u.a. Boeckmann 1994; Hipfl 1996; Tulodziecki 1998; Schludermann 2002). Desweiteren gehen sie von der emotionalen Teilhabe am Medienhandeln aus: Demnach biete erst die subjektive Auseinandersetzung die Möglichkeit, Medieninhalte neu zu bewerten und damit zu einer selbstbestimmten Veränderung in der Mediennutzung zu führen (vgl. Boeckmann 1994; Hipfl 1996).

\section{Digitale Ungleichheiten}

Ausgehend von einem demokratietheoretischen Zugang zu Partizipation spielen die Forschungsergebnisse der Medien- und Kommunikationsforschung zur «digitalen Ungleichheit» (Engl. «Digital Divide») eine besondere Rolle. Einerseits thematisieren sie einen ungleichen Zugang zu Kommunikationstechnologien, andererseits 
ungleiche Nutzungsweisen mit Medien (Second Digital Divide) ${ }^{1}$. Demnach sind insbesondere Alter, Geschlecht und Bildung für die Form der Mediennutzung und für die Teilhabe an einer mediatisierten Gesellschaft bedeutsam. Fähigkeiten, die in Zusammenhang mit Medien(-nutzung) relevant sind und durch Medien angeeignet werden, sind demnach unterschiedlich ausgeprägt: Sie tragen in unterschiedlicher Weise zu Bildungsprozessen und zur Realisierung gesellschaftlicher Teilhabemöglichkeiten bei. Somit spiegeln sich soziale Ungleichheiten nicht nur in der Mediennutzung wieder, sie werden durch Fehlannahmen bzgl. Geräteausstattung, Medienkompetenzen etc. in ihr auch reproduziert (vgl. Iske et al. 2007; Tillmann 2008; Klein 2008).

\section{Lern- und Bildungskontexte}

Die Kommunikationsanlässe, die die Medienpädagogik schaffen möchte, sind abhängig von den Kontexten, innerhalb derer sie verortet sind. Zudem werden sie beeinflusst von den Fach- bzw. Praxiscommunities, in denen sie beheimatet sind. So betrachtet die Medienpädagogik insgesamt sowohl formale Bildungskontexte wie Schulen und Hochschulen als auch non-formale Bildungskontexte und informelle Bildungsgelegenheiten wie das Wechselverhältnis von Subjekt, Medien und Familie. In der Mediendidaktik - verstanden als Lehren und Lernen mit Medien als Teilbereich der Medienpädagogik ${ }^{2}$ - wird oft nur der Ausschnitt formalisierten Lernens mit Medien betrachtet, sodass im gleichen Atemzug verwandte Begriffe wie mediengestütztes Lernen oder E-Learning genannt werden. In der Medienpädagogik hingegen werden vielfach non-formale Bildungskontexte und informelle Bildungsgelegenheiten in den Blick genommen, wie sie z.B. in der ausserschulischen und offenen Jugendarbeit zu finden sind. Mediendidaktische Begriffe und damit zusammenhängende Konzepte sind für die jüngeren Entwicklungen in der Erwachsenenbildung von Bedeutung, sie stellen allerdings nur einen Teil medienpädagogischer Praxis dar. Darüber hinaus ergeben sich konzeptionelle Engfassungen, wenn primär auf bildungstechnologische Konzepte in der Erwachsenenbildung zurückgegriffen wird.

1 Ausschlaggebend für die Diskussion um ungleiche Nutzungsweisen war eine empirische Studie von Hargittai (2002), die Unterschiede bei den «Online Skills» von Personen zwischen 18 und 81 untersucht hat. Bei Kindern und Jugendlichen lassen sich jedoch nur noch wenige Ungleichheiten beim Zugang zu digitalen Medien begründen (vgl. MPFS 2016). Seither werden mit Fokus auf Kindern und Jugendlichen unterschiedliche Nutzungsweisen von Jugendlichen gezeigt und problematisiert (vgl. u.a. Otto/Kutscher 2004; Brüggen/Wagner 2008; Henrichwark 2009; Niesyto et al. 2010). Erwachsene hingegen geraten erst langsam ins (nicht nur empirische) Blickfeld.

2 Eine Ausführung des Diskurses zur Positionierung der Mediendidaktik zur Medienpädagogik und zur allgemeinen Didaktik findet sich in Süss et al. (2010). 


\section{Handlungsorientierung}

Das Prinzip der Handlungsorientierung ist vielen medienpädagogischen Konzepten inhärent. Es zielt darauf ab, zum eigenen Handeln mit und durch Medien und zum sozial verantwortlichen Handeln anzuregen. Dabei liegt der Fokus auf der Förderung von Reflexion und Handlungsoptionen ohne festgelegte Handlungsziele. Sprich, es geht vorwiegend um die Ermöglichung von Medienbildung, weniger um das Erreichen einer spezifischen Kompetenz im Sinne einer Performanz ${ }^{3}$. Diese Unterscheidung ist insbesondere in den unterschiedlichen Lesarten von Medienkompetenz(en) bedeutend: Darin wird ersichtlich, dass ein zunächst pädagogisch gemeinter Begriff zunehmend von einer qualifikatorisch geprägten Kompetenzdebatte eingeholt und vorwiegend technisch-funktional verstanden wird. Dabei steht das Handeln innerhalb medienpädagogischer Praxis - gerade in der aktiven Medienarbeit (vgl. Schell 1989) - oft für sich und wird erst angesichts subjektiver Sinnzuschreibung «gut». Missverständlich ist mitunter, dass Handlungsorientierung innerhalb formal geplanter Bildung als problemorientiertes Lernen umgesetzt wird. Mit der Problemorientierung geht dann eine Engführung in Bezug auf Lernen und Bildungsformate einher, die durch den formalen Rahmen der Bildungseinrichtung bestimmt und mit der prinzipiellen Offenheit medienpädagogischer Konzepte allenfalls noch verwandt ist.

\section{Medienbegriff}

Für die Medienpädagogik ist schliesslich die Annahme zentral, dass der (Bildungs-) Kontext, innerhalb dessen subjektives Handeln stattfindet, ähnlich prägend ist wie die Wahl der Medien selbst: Anders als vielfach öffentlich angenommen (vgl. Schiefner-Rohs/Hofhues, 2017), geht die Medienpädagogik zunehmend von «starken» Medien aus, ohne zugleich eine direkte, sprich kausale Medienwirkung vorauszusetzen (vgl. Meyer 2008; zum Raumdiskurs in der Medienpädagogik vgl. u.a. Tillmann 2008; Tully 2009). Hintergrund ist, dass Medien in der Medienpädagogik als technische Gebrauchsmedien und ergänzend als «Struktur und Situation» (Krotz 2012, 44) verstanden werden. In letzterer Perspektive ermöglichen und modifizieren Medien Kommunikation in Form von «transformierten Texten in Bezug auf, aber auch in Konkurrenz zur Face-to-Face-Kommunikation, ferner mit Bildern und Tönen, [...] und unter Verwendung verschiedener Symbolsysteme.» (ebd.) Auch in der Erwachsenenbildung werden Medien seit den 1980er Jahren als Werkzeug/Instrument und als Gegenstand/Inhalt verstanden (vgl. Faulstich 1986; Faulstich/Faulstich-Wieland 1988; Hüther 1993). Programmatische Schriften von Bundesministerien, Stiftungen etc. gehen allerdings davon aus, dass Informations- und Kommunikationstechnologien (IKT) eine steigende Bedeutung für die Teilhabe am gesellschaftlichen und kulturellen

3 Ursprung: Chomsky, Noam. 1973. Sprache und Geist, (Erstauflage: 1968). Frankfurt: Suhrkamp 
Leben einnehmen und speziell der Arbeitsmarkt einen wachsenden Bedarf an Fachkräften entwickelt, die mit IKT «kompetent» umgehen müssen und sollen.

Anzufügen ist, dass neben den sechs skizzierten Leitideen zwei Ansätze grundlegend innerhalb der Medienpädagogik diskutiert werden. Gemeint sind Ansätze zur Förderung von Medienkompetenzen und Medienbildung, die kein Gegensatzpaar bilden, sondern - wie Hugger (2008) es beschreibt - «zwei Seiten der Medaille» (ebd., 97) sind und die skizzierten Leitideen aufnehmen. Diese Unterschiedlichkeit zu registrieren, ist auch für die Praxis der Erwachsenenbildung zentral, denn innerhalb beider Ansätze werden «je eigene Fragen und Probleme einer gesellschaftlichen Realität, die in hohem Masse durch Medien geprägt wird, zugänglich und bearbeitbar» (Fromme/Jörissen 2010, 47) gemacht:

Die Wurzeln des Medienkompetenz-Begriffs liegen in der Habilitationsschrift «Kommunikation und Kompetenz» von Baacke (1980). Nach Baacke differenziert sich Medienkompetenz in vier Dimensionen: 1) Medienkritik, 2) Medienkunde, 3) Mediennutzung und 4) Mediengestaltung. In der Diskussion um die Medienkompetenz als Zielwert der Medienpädagogik wurden weitere Dimensionierungen des Begriffs vorgenommen (vgl. ausführlich Gapski 2001), nicht zuletzt vor der Frage, wie sich in anderen Bindestrichdisziplinen der Pädagogik Medienkompetenz herleiten lässt. So verknüpften Dewe und Sander (1996) den Begriff der Medienkompetenz mit dem Kompetenzdiskurs der Erwachsenenbildung, indem sie das Modell der Schlüsselqualifikation mit dem Modell der Medienkompetenz zusammenführen. Innerhalb der Erwachsenbildung werden unterschiedliche Medienkompetenzmodelle rezipiert (von Hippel 2007, 67f.). Allerdings zeichnen sich die Medienkompetenz-bezogenen Diskursstränge ihrer Ansicht nach durch einen normativ-medienpädagogischen und einen wirtschaftlich-medientechnischen Diskurs aus. Das wirtschaftliche-technische Verständnis betont die Förderung spezifischer Kenntnisse mit Blick auf einen beruflichen Bereich und findet sich innerhalb der Medienpädagogik eher in der Mediendidaktik wieder. Der normativ-medienpädagogische Diskurs fokussiert hingegen die Bedeutung von Bildung für die Entwicklung eines Menschen und ist eher mit der Allgemeinbildung assoziiert. Innerhalb der Medienpädagogik ergibt sich deshalb eine Nähe zum Verständnis von Medienbildung: Im Zentrum des Medienbildungsbegriffs steht die subjektive Bereitschaft zur Veränderung, die es Menschen ermöglicht, die Welt (und sich selbst) zu sehen und wahrzunehmen, sodass sie in einer komplexeren Welt mit weniger vorhersehbaren Biographien und Karrieren zurechtkommen, Orientierung gewinnen und sich zu dieser Welt kritisch-partizipativ verhalten. Anfangs als Gegenbegriff zur Medienkompetenz eingeführt, kritisieren Vertreterinnen und Vertreter des Medienbildungsbegriffs (u.a. Aufenanger 2000; Marotzki 2004; Jörissen/ Marotzki 2009) die Vermittlung von Fakten- bzw. Verfügungswissen. So würden eher Qualifikationsziele verfolgt (vgl. ausführlich Hugger 2008, 96f.). Nicht zuletzt deshalb laden wir im Folgenden zu einer interdisziplinären Perspektivübernahme und zur 
Adaption der vielfältigen medienpädagogischen Leitideen und Ansätze ein, erweisen sich alle gemeinsam als fruchtbar für den Diskurs um Medien in der Erwachsenenbildung.

\section{Praxis der medienpädagogischen Erwachsenenbildung}

Vorweggenommen sei, dass bei Betrachtung der aktuellen wirtschaftlich-politischen Forderungen, der Leitideen der Medienpädagogik sowie der erwachsenenpädagogischen Praxis, Disparitäten zwischen der erwachsenenpädagogischen und der medienpädagogischen Verortung öffentlich beschworen werden. Im Fokus der öffentlichen Debatte stehen vor allem wirtschaftliche und industrielle Anforderungen des digitalen Wandels, die unter den Stichworten «Wirtschaft 4.0» und «Industrie 4.0» firmieren. Erwachsenenbildung wird dabei vorwiegend als berufliche Bildung verstanden und medienpädagogische Aspekte der Allgemeinbildung, wie sie von Hippel (2011) als Handlungspraktiken in der medienpädagogischen Erwachsenenbildung beschreibt, kommen sukzessive abhanden, mehr noch: Mit Blick auf das Aufkommen digitaler Medien und mediendidaktischer Konzepte zeichnet sich die Stärkung der wirtschaftlichen-medientechnischen Perspektive bzw. einer technisch-instrumentellen Definition von Medienkompetenzen ab. Wie vielfältig sich medienpädagogische Praxis in der Erwachsenenbildung prinzipiell darstellen könnte, wird im Folgenden anhand exemplarischer Bereiche skizziert.

\section{(Digitale) Technologien zur Unterstützung selbstorganisierten Lernens}

Online-Coaching, Lernvideos, Diskussionsforen, computerbasierte Testverfahren oder MOOCs (Massive Open Online Courses) sind Ausdruck für die wachsende Forderung zur Einbindung (digitaler) Medien in die Angebotsstrukturen rund um «Adult Learning». Insbesondere auf EU-Ebene (European Commission 2015, 53) wird die stärkere Einbindung digitaler Technologien in der Erwachsenenbildung gefordert (vgl. Kerres 2013, 412, Pietraß 2015, 5). Diese weitestgehend online basierten Bildungsangebote können als Erweiterung bzw. Intensivierung mediendidaktischer Konzepte in der Erwachsenenbildung beschrieben werden. Das digitale Kursformat eines MOOCs verzichtet vollständig auf Präsenzveranstaltungen und nutzt ausschliesslich mediale Formen der Wissensvermittlung und Kommunikation. Durch die für viele digitale Lernformen charakteristische orts- und zeitentgrenzten Angebotsform können grosse Menschenmengen an den Bildungsinhalten teilhaben. Dass MOOCs in der Erwachsenenbildung - wenn auch langsam - an Relevanz gewinnen, zeigt die Plattform «Volkshochschul-Informationen» ${ }^{4}$, die das Thema umfassend aufgreift und konstatiert, dass MOOCs «in gewissermassen eine neue Ära an den Volkshochschulen

4 http://www.vhs.info/mooc. 
eingeläutet [haben]» (Volkshochschulinformationen, online). Auf den ersten Blick werden dort die Teilhabemöglichkeiten digitaler und mobiler Medien als Ressource erkannt, um den Zugang zu Bildung zu erleichtern und somit die Weiterbildungsbeteiligung zu erhöhen. So fordert auch der Deutsche Volkshochschulverband (2015) in einer Resolution eine «digitale Weiterbildungsoffensive» (ebd., 1), um Menschen zu erreichen, «die bisher nicht ausreichend am lebenslangen Lernen partizipieren können» (ebd.) und aus individuellen oder strukturellen Gründen «kaum Chancen haben, an Präsenzlernangeboten teilzunehmen» (ebd.). Auf den zweiten Blick stellt sich die Bearbeitung sozialer und digitaler Ungleichheiten in Form von Online-LernAngeboten als widersprüchlich dar. Es soll Ungleichheiten im Zugang zu Bildungsangeboten durch die Allgegenwart digitaler Technologien entgegengewirkt werden, zugleich nehmen Ungleichheiten im Zugang zu. Auch die Nutzung digitaler Medien bringt neue Exklusionsfaktoren mit (Klein 2008; 2015). So ist festzuhalten, dass MOOCs mehrheitlich von Menschen genutzt werden, die bereits über eine höhere Bildung verfügen (Schulmeister 2013, 26f.). Abstrahiert man vom Beispiel, finden sich vor allem Hinweise darauf, wie Partizipation in der Erwachsenenbildung jüngst gelesen wird. Es wird deutlich, dass weniger Partizipation an Gesellschaft, sondern eher selbstgesteuertes Lernen für weiterbildungsinteressierte, Menschen, d.h. eine spezifische erwachsene Zielgruppe, gefördert wird.

\section{Förderung technisch-funktionaler Medienkompetenzen bei Erwachsenen}

Sollen Medienkompetenzen in der Erwachsenenbildung gefördert werden, finden sich in der Praxis zudem eine Vielzahl problemorientierter Konzepte, mit der auf die technisch-instrumentellen Anforderungen des Arbeitsmarkts reagiert wird. Beispielhaft angeführt kann hier der Europäische Computerführerschein ECDL ${ }^{5}$, der als «internationaler Standard für Digitale Kompetenz» den Fokus auf prüfbare Computerkenntnisse legt. Werden gegenwärtige Forderungen und Bestrebungen zur Erwachsenenbildung und digitalen Technologien in den Blick genommen, wird diese Annahme sogar noch verstärkt. Es fallen vor allem Zielbeschreibungen zur individuellen Kompetenzentwicklung rund um digitale Technologien auf EU-Ebene auf «to strengthen human capital, employability und competitiveness» (vgl. European Commission 2016). Insbesondere der digitale Wandel in der Wirtschaft und der Wandel der Arbeitswelt werden als Herausforderungen betont (vgl. ebd., 2). Die Arbeitsgruppe stellt fest, dass sich die technisch-qualifikatorischen Anforderungen und die Abdeckung und Qualität entsprechender Weiterbildungsangebote als disparat darstellen (vgl. ebd., 3).

Diese Bestrebungen der EU-Mitgliedsstaaten bis Mitte 2017 spiegeln sich im öffentlichen Diskurs im deutschsprachigen Raum wieder. Deutlich wird einerseits der

5 http://www.ecdl.de. 
(verkürzte) Rekurs auf Informations- und Kommunikationstechnologien. Andererseits wird in der Agenda der Europäischen Kommission vor allem problemorientiert argumentiert. Wie angeführt, geht damit eine Engführung in Bezug auf Lernen und Bildungsformate einher, die durch den formalen Rahmen der Bildungseinrichtung bestimmt werden. Kommunikationsanlässe ausserhalb formaler Bildungseinrichtungen zu schaffen, was mittels Handlungsorientierung angestrebt wird, ist in dieser Lesart zweitrangig. ${ }^{6}$ Schon die etwas älteren empirischen Befunde der institutionenübergreifenden Programmanalyse von 676 medienbezogenen Veranstaltungen an insgesamt sechs Institutionen der Erwachsenenbildung (von Hippel 2007, 696f.) weisen diesen Massnahmenschwerpunkt auf: Im Vordergrund der Programme steht die instrumentell-qualifikatorische Medienkunde und der gesellschaftliche Bedarf nach Angeboten zum technischen Umgang. Bemerkenswert ist, dass die Förderung der instrumentell-qualifikatorischen Medienkunde in Kombination mit der Mediengestaltung und die informative Medienkunde in Kombination mit der Medienkritik auftritt (vgl. ebd., 698). Von Hippel konstatiert, dass sich in der Erwachsenenbildung zwei Linien der Medienpädagogik widerspiegeln würden: «die Tradition der Förderung von Partizipation durch Mediengestaltung und die Förderung eines kritischen Umgangs mit Medien aufgrund von Wissen über sie.» (ebd.). Gemeinsame Orientierungen von Medienpädagogik und Erwachsenenbildung werden darin deutlich. So liegen wie in der Medienpädagogik die historischen Wurzeln der Erwachsenenbildung in der Aufklärung, deren Impetus und Ziel der Mündigkeit heute handlungsleitend für viele Bindestrichpädagogiken ist (vgl. Nuissl 2011, 330). Vor diesem Hintergrund sind die Orientierung an Mündigkeit und gesellschaftlicher Teilhabe in der Erwachsenenbildung handlungsleitend. Inwieweit Medienpädagogik und Erwachsenenbildung gemeinsame Begriffsverständnisse haben und Konzepte teilen, ist bislang allerdings wenig aufgearbeitet.

\section{Qualifizierung von Erwachsenenbildnerinnen und -bildnern für den Medienwandel} Schliesslich rücken die fachlichen Akteurinnen und Akteure der Erwachsenenbildung, die sich in dem Gefüge zwischen Subjekt, Medien und Bildung befinden, und die Organisationen der Erwachsenenbildung selbst in den Blick. Für pädagogische Fachkräfte werden Wissen und Fähigkeiten in der Mediendidaktik bedeutsam; gleichzeitig stehen sie selbst vor der Auswahlentscheidung für/gegen «richtige» Vermittlungskonzepte. Die Reflexion der Ansätze zur Förderung von Medienkompetenzen und Medienbildung gehört für sie ebenso dazu wie ihr eigener Umgang mit der

6 Als Beispiel eignet sich das Quantified Self zur Thematisierung unter Erwachsenen. Insgesamt spiegelt sich ein umfassender Rekurs auf medienpädagogische Konzepte im fachlichen und politischen Diskurs der Erwachsenenbildung kaum wieder. Bestätigt wird diese These in der politischen Agenda, die sich in den angeführten Kompetenzanforderungen u.a. auf akute Aspekte der Digitalisierung und Datafizierung bezieht, während selbstreflexive Lern- und Orientierungsprozesse zwischen Subjekt - Medien - Bildung weniger thematisiert werden. 
Unbestimmtheit (digitaler) Medien bzw. des Medienhandelns. Es scheint folgerichtig, in der Erwachsenenbildung den Ansatz zur Förderung «Medienpädagogischer Kompetenzen» aufzunehmen, der in der Lehrpersonenbildung etabliert ist und das notwendige Wissen sowie anvisierte Fähigkeiten von Lehrenden beschreibt, um Schülerinnen und Schülern die (Weiter-)Entwicklung von Medienkompetenzen zu ermöglichen (vgl. Blömeke 2000; Tulodziecki 2012). Wird den wissenschaftlichen Begründungen zur medienpädagogischen Kompetenz gefolgt, wird vor allem der individuellen Medienkompetenz und - so ist zu erweitern - der Medienbildung der Professionellen eine besondere Rolle zugesprochen, da der Kompetenzbegriff auf die Handlungsfähigkeit in einem bestimmten Handlungsfeld zielt (vgl. Tulodziecki 2012, S. 275f.) Medienpädagogische Kompetenzen sind entsprechend in Kompetenzbeschreibungen der Erwachsenenbildung zu finden, sie sind allerdings unterschiedlich ausgeprägt und werden unterschiedlich gewichtet sowie nicht in allen Tätigkeitsbereichen als Querschnittsthema berücksichtigt (Rohs et al. 2017).

Die Förderung von Medienkompetenzen und Medienbildungsprozessen in der Erwachsenenbildung stellt sich so zunehmend als diskursives Spannungsfeld dar, in dem sich sowohl die professionellen Akteurinnen und Akteure als auch die Organisationen innerhalb und ausserhalb der Erwachsenenbildung verorten. Gleichermassen sind die diversen Bildungskontexte selbst Teil des gesellschaftlichen und kulturellen Wandels und gestalten diesen mit.

\section{Antinomien in der Praxis der medienpädagogischen Erwachsenenbildung}

Die ökonomisch geprägten Debatten um die Digitalisierung haben Auswirkungen auf die Erwachsenenbildung. Wie exemplarisch dargelegt wurde, werden sowohl auf Ebene der Erwachsenenbildnerinnen und -bildner als auch auf Ebene der Adressatinnen und Adressaten von Angeboten der Erwachsenenbildung vor allem technischinstrumentelle Kompetenzbereiche als Anforderungen und Zielsetzungen formuliert. So wird unter Medienpädagogik in der Erwachsenenbildung vor allem die Nutzung von digitalen Technologien zur Vermittlung von Inhalten verstanden; somit eine mediendidaktische Kompetenz betont. Auf der anderen Seite lassen sich deutliche Parallelen in den Leitideen und den Zielsetzungen zwischen Medienpädagogik und Erwachsenenbildung finden, die die Möglichkeiten eines interdisziplinären Diskurses betonen. Dennoch zeigen sich vor allem Disparitäten zwischen dem Medienbezug in der Erwachsenenbildung und dem Diskurs der Medienpädagogik. Technologie-basierte Medien spielen in der Erwachsenenbildung seit jeher eine besondere Rolle, vor allem sind es aber «Angebote im Umgang mit dem Computer, die in der Weiterbildung Einzug hielten» (Stang 2003, 11). Stang verweist in dieser Kritik insbesondere auf Baacke, der das gesamte Bildungsspektrum im Zuständigkeitsbereich der Medienpädagogik verortet. 
Von Hippel $(2007 ; 2011)$ begründet die technisch-instrumentelle Orientierung in der erwachsenenpädagogischen Medienkompetenzförderung mit den Herausforderungen des Programmplanungshandelns im Spannungsfeld heterogener Erwartungen. So besteht zwar historisch keine ausschliessliche Kundenorientierung in der Erwachsenenbildung, dennoch bestehen Antinomien zwischen den pädagogischen Zielen - hier einer umfassenden Medienpädagogik - und ökonomischen Kriterien, insbesondere dann, wenn die Interessen von Adressatinnen und Adressaten, wirtschaftlichen, politischen, wissenschaftlichen und pädagogischen Akteurinnen und Akteuren divergent sind und diese ausbalanciert werden müssen (vgl. von Hippel 2011, 51f.).

\section{Medien zur selbst- und mitverantwortlichen Teilhabe von Erwachsenen an Alltag} und Gesellschaft (Fazit)

Eingangs haben wir zwei Fragen formuliert, die abbilden sollten, welche Leitideen des medienpädagogischen Diskurses für die Erwachsenenbildung besonders relevant sind und welche sich in der erwachsenenbildnerischen Praxis wiederfinden, denn: Mit Grell (2017) benötigt es für alltägliches und gesellschaftspolitisches Handeln Kenntnisse und Fähigkeiten, um die Prozesse und die Bedeutung digitaler Informationen und Kommunikation «zu verstehen, sich darin orientieren zu können und eine digitalisierte Welt mitgestalten zu können.» (ebd., 83). Somit ist bei der Integration von Medien in die Erwachsenenbildung kritisch zu hinterfragen, «inwieweit sie neue Gestaltungsräume für Erwachsene eröffnen oder aber lediglich neue Anpassungszwänge bedienen» (ebd.). Neben dem umfangreichen Blick auf vorliegende Leitideen, Ansätze und (exemplarische) Konzepte aus Medienpädagogik und Erwachsenenbildung halten wir auch die systematische Bezugnahme zum Verständnis von Medienbildung für zentral.

In unseren Betrachtungen haben wir Hinweise darauf gefunden, dass Medienpädagogik in der Erwachsenenbildung vielfach als Mediendidaktik übersetzt wird. Sowohl in der politischen Agenda als auch in der Qualifizierung von Erwachsenenbildnerinnen und -bildnern wird die stärkere Einbindung digitaler Technologien forciert, allerdings vielfach ohne explizit auf medienpädagogische Ideen und Ansätze zurückzugreifen. Daran knüpft eine Perspektive von Partizipation an, die ein selbstgesteuertes/-organisiertes Lernen betont und sozialen Ungleichheiten entgegenwirken soll. Wie empirische Studien zeigen, profitieren von Online-Angeboten allerdings vor allem Menschen, die bereits einen hohen Bildungsstand vorweisen können. Eine Verkürzung von Medienpädagogik als Mediendidaktik läuft somit Gefahr, soziale und digitale Ungleichheiten zu verstärken, statt ihnen entgegen zu wirken. Nicht ohne Grund verzichten die meisten Alphabetisierungskonzepte derzeit auf digitale Medien. Weiterhin finden sich Hinweise darauf, dass Medienkompetenzen und Medienbildung in der Erwachsenenbildung vor dem Hintergrund arbeitsmarkbezogener 
Problemstellungen in den Blick genommen werden. Insbesondere die Medienkunde, das Wissen über Medien und Mediensysteme, wird in der Erwachsenenbildung gefördert, während eine Thematisierung selbstbestimmten Handelns unter den Bedingungen des digitalen Wandels vielfach ausbleibt (vgl. von Hippel 2007, 697).

Es schliesst sich die Frage an, welchen Nutzen eine konsequente medienpädagogische Perspektive für die (weitere Professionalisierung der) Erwachsenenbildung haben könnte. Hierbei knüpfen wir an die Forderung zum medienpädagogischen Perspektivwechsel von Allert und Richter (2016) an: Ausgehend von der praxistheoretischen Sichtweise, dass sich das Selbst im relationalen Gefüge von Menschen, Technologien und Sozialem konstituiert und im Sinne der «Kultur der Digitalität» (Stadler 2016) kulturelle Formen, Institutionen und Gewissheiten erodieren, fordern Allert und Richter (2016, 9f.) eine Neufassung der Vorstellungen von Medienbildung und Medienkompetenzen und - so ist zu erweitern - der Förderung entsprechender Lernund Bildungsprozesse. Medienbildung, verstanden als Transformation menschlicher Lebensorientierung in Auseinandersetzung mit konjunktiven Transformationsräumen, erfordert die gestaltende und produktive Auseinandersetzung mit der Unbestimmbarkeit digitaler Kulturen und setzt immer kreative Praktiken voraus. Entsprechend bedarf Medienbildung «eines Verständnisses von Kompetenz, das sich nicht in der Verfügbarkeit instrumenteller Fähigkeiten erschöpft.» (ebd., 10).

Für die Erwachsenenbildung ist daraus abzuleiten, dass sie vermehrt als Erwachsenenbildung in einer digitalen Kultur zu verstehen ist, die ihre Rolle in der Relationierung von Subjekt, Medien und Bildung reflektiert. Die Leitideen der Medienpädagogik können dabei eine Orientierung sein und vor einer Instrumentalisierung erwachsenenpädagogischer Bildungsorte schützen. Daran anknüpfend bedeutet medienpädagogische Erwachsenenbildung eine Förderung hin zu einer selbst- und mitverantwortlichen Teilhabe des Subjekts an der digitalen Gesellschaft und Kultur. Eine Stärkung dieser Perspektive stellt ein deutliches Potenzial für die medienpädagogische Forschung und Praxis in der Erwachsenenbildung dar.

\section{Literatur}

Allert, Heidrun, und Christoph Richter. 2016. Kultur der Digitalität statt digitaler Bildungsrevolution. Arbeitspapier. http://nbn-resolving.de/urn:nbn:de:0168-ssoar-47527-7.

Aufenanger, Stefan. 2000. «Medienpädagogik im 21. Jahrhundert. Kernpunkte einer pädagogischen Zukunftsforschung». In Spannungsfeld Medien und Erziehung: Medienpädagogische Perspektiven, hrsg. v. Hubert Kleber, 33-44. München.

Baacke, Dieter. 1980. Kommunikation und Kompetenz. Grundlegung einer Didaktik der Kommunikation und ihrer Medien. München, Weinheim: Juventa.

Blömeke, Sigrid. 2000. Medienpädagogische Kompetenz: theoretische und empirische Fundierung eines zentralen Elements der Lehrerausbildung. München: kopaed. 
Boeckmann, Klaus. 1994. «Thema: Medienmündigkeit (1)». medienpraktisch, 1/1994, 34-37.

Brüggen, Niels, und Ulrike Wagner. 2008. «Pädagogische Konsequenzen.» In Medienhandeln in Hauptschulmilieu Mediale Interaktion und Produktion als Bildungsressource, hrsg. v. Ulrike Wagner, 223-246. München: kopaed.

Deutscher Volkshochschulverband. 2015. Resolution. Volkshochschulen fordern digitale Weiterbildungsoffensive. https://www.dvv-vhs.de/fileadmin/user_upload/6_Themenfelder/ Erweiterte_Lernwelten/Resolution_MV.pdf.

Dewe, Bernd, und Uwe Sander. 1996. «Medienkompetenz und Erwachsenenbildung». In Medienkompetenz als Schlüsselbegriff, hrsg. v. Antje von Rein, 125-142. Bad Heilbrunn.

European Commission. 2015. Education and Training 2020. Improving Policy and Provision for Adult Learning in Europe. http://ec.europa.eu/dgs/education_culture/repository/education/library/reports/policy-provision-adult-learning_en.pdf.

European Commission. 2016. A new Skills Agenda for Europe. Working together to strenghen human capital, employability and competitiveness. http://ec.europa.eu/social/BlobServle $\mathrm{t}$ ?docld=15621\&langld=en.

Faulstich, Peter, und Hannelore Faulstich-Wieland. 1988. Computer - Kultur. Erwartungen Ängste - Handlungsspielräume. München: Lexika.

Faulstich, Peter. 1986. Betriebliche Weiterbildung. Neue Technik - Neue Produktionskonzepte. Kassel: Grimm.

Fromme, Johannes, und Benjamin Jörissen. 2010. «Medienbildung und Medienkompetenz. Berührungspunkte und Differenzen nicht ineinander überführbarer Konzepte». merz - Medien + Erziehung 5/2010, 46-54.

Gapski, Harald. 2001. Medienkompetenz. Eine Bestandsaufnahme und Vorüberlegungen zu einem systemtheoretischen Rahmenkonzept. Wiesbaden: Westdeutscher Verlag.

Grell, Petra. 2017. «Erwachsenenbildung». In Grundbegriffe Medienpädagogik, hrsg. v. Bernd Schorb, Anja Hartung, und Christine Dallmann, 79-83. München: kopaed.

Hargittai, Eszter. 2002. "Second-Level Digital Divide: Differences in People's Online Skills». First Monday, 4/2002. http://dx.doi.org/10.5210/fm.v7i4.942.

Henrichwark, Claudia. 2009. Der bildungsbezogene mediale Habitus von Grundschulkindern: Eine empirische Studie zur Reproduktion sozialer Ungleichheit in der Schule und Familie. Wuppertal (Dissertation). http://elpub.bib.uni-wuppertal.de/edocs/dokumente/fbg/paedagogik/diss2009/henrichwark/dg0901.pdf.

Hepp, Andreas, Matthias Berg, und Cindy Roitsch. 2014. Mediatisierte Welten der Vergemeinschaftung. Kommunikative Vernetzung und das Gemeinschaftsleben junger Menschen. Wiesbaden: VS Verlag für Sozialwissenschaften.

Herber, Erich, Bernhard Schmidt-Hertha, und Sabine Zauchner-Studnicka. 2013. «Erwachsenen- und Weiterbildung. Technologieeinsatz beim Lernen und Lehren mit Erwachsenen». In L3T. Lehrbuch für Lernen und Lehren mit Technologien, hrsg. v. Martin Ebner und Sandra Schön. http://l3t.tugraz.at/index.php/LehrbuchEbner10/article/view/133. 
Hipfl, Brigitte. 1996. «Medienmündigkeit und Körpererfahrung». medienpraktisch, 3/1996, 32 35.

Hippel, Aiga von. 2007. Medienpädagogische Erwachsenenbildung. Eine Analyse von pädagogischen Auftrag, gesellschaftlichen Bedarf und Teilnehmendeninteressen. Schriftenreihe der Landesmedienanstalt Saarland. Band 14. Saarbrücken: LMS.

Hippel, Aiga von. 2011. «Erwachsenenbildung und Medien». In Handbuch Erwachsenenbildung/ Weiterbildung, 5. Auflage, hrsg. v. Rudolf Tippelt, und Aiga von Hippel, 687-706. Wiesbaden Springer VS.

Hugger, Kai-Uwe. 2008. «Medienkompetenz». In Handbuch Medienpädagogik, hrsg. v. Uwe Sander, Friederike von Gross, und Kai-Uwe Hugger, 93-99. Wiesbaden: VS Verlag für Sozialwissenschaften.

Hüther, Jürgen. 1993. «Medienpädagogik zwischen politischer und informationstechnischer Bildung». In Internationales Jahrbuch der Erwachsenenbildung, hrsg. v. Joachim. H. Knoll. Köln, Weimar, und Wien. Böhlau.

Hüther, Jürgen. 1994. «Medienpädagogische Konzepte in der Bildungsarbeit mit Erwachsenen. Historische und aktuelle Aspekte der Kooperation von Medienpädagogik und Erwachsenenbildung». In Handbuch der Medienpädagogik. Theorieansätze - Traditionen - Praxisfelder - Forschungsperspektiven, hrsg. v. Susanne Hiegemann, und Wolfgang H. Swoboda, 289-301. Opladen: Leske+Budrich.

Iske, Stefan, Alexandra Klein, Nadia Kutscher, und Hans-Uwe Otto. 2007. «Virtuelle Ungleichheit und informelle Bildung: Eine empirische Analyse der Internetnutzung Jugendlicher und ihre Bedeutung für Bildung und gesellschaftliche Teilhabe». In Grenzenlose Cyberwelt?: Zum Verhältnis von digitaler Ungleichheit und neuen Bildungszugängen für Jugendliche, hrsg. v. Kompetenzzentrum Informelle Bildung, 65-92. Wiesbaden: Springer VS.

Jörissen, Benjamin, und Winfried Marotzki. 2009. Medienbildung - Eine Einführung. Stuttgart: UTB.

Kerres, Michael. 2013. Mediendidaktik. Konzeption und Entwicklung mediengestützer Lernangebote. 4. Aufl. München: Oldenbourg

Klein, Alexandra. 2008. Soziales Kapital Online. Soziale Unterstützung im Internet. Eine Rekonstruktion virtualisierter Formen sozialer Ungleichheiten. Dissertation an der Universität Bielefeld. https://pub.uni-bielefeld.de/download/2301811/2301814.

Knauer, Reingard, und Benedikt Sturzenhecker. 2005. «Partizipation im Jugendalter». In Kinder- und Jugendpartizipation. Im Spannungsfeld von Interessen und Akteuren, hrsg. v. Benno Hafeneger, Mechthild. M. Jansen, und Torsten Niebling, 63-94. Opladen.

Krotz, Friedrich. 2007. Mediatisierung: Fallstudien zum Wandel der Kommunikation. Wiesbaden.

Krotz, Friedrich. 2012. «Von der Entdeckung der Zentralperspektive zur Augmented Reality: Wie Mediatisierung funktioniert.» In Mediatisierte Welten: Forschungsfelder und Beschreibungsansätze, hrsg. v. Friedrich Krotz, und Andreas Hepp, 27-55. Wiesbaden: Springer VS.

Marotzki, Winfried. 2004. «Von der Medienkompetenz zur Medienbildung». In Lebensbegleitendes Lernen als Kompetenzentwicklung. Analysen, Konzeptionen, Handlungsfelder, hrsg. v. Rainer Brödel, und Julia Kreimeyer, 63-74. Bielefeld: Bertelsmann. 
Meyer, Torsten. 2008. «Zwischen Kanal und Lebens-Mittel: pädagogisches Medium und mediologisches Milieu». In Pädagogische Medientheorie, hrsg. v. Fromme, Johannes, und Werner Sesink, 71-94. Wiesbaden: VS Verlag für Sozialwissenschaften.

MPFS (Medienpädagogischer Forschungsverbund Südwest). 2016. JIM-Studie 2016: Jugend, Information, (Multi-) Media. Basisstudie zu Medienumgang 12-bis 19-Jähriger in Deutschland. Stuttgart. https://www.mpfs.de/fileadmin/files/Studien/JIM/2016/JIM_Studie_2016.pdf.

Niesyto, Horst, Dorothee M. Meister, und Heinz Moser, Hrsg. 2010. «Medien und soziokulturelle Unterschiede». MedienPädagogik. Zeitschrift für Theorie und Praxis der Medienbildung (Themenheft Nr. 17). http://dx.doi.org/10.21240/mpaed/17.X.

Nuissl, Ekkehard. 2011. «Ordnungsansätze der Erwachsenenbildung in Deutschland». In Handbuch Erwachsenenbildung/Weiterbildung, 5. Auflage, hrsg. v. Rudolf Tippelt, und Aiga von Hippel, 329-246. Wiesbaden: VS Verlag für Sozialwissenschaften.

Olbermann, Samuel. 2016. «Industrie 4.0 - Chance oder notwendiges Übel für kirchliche Erwachsenenbildung?». Forum Erwachsenenbildung 3/2016, 47-48.

Otto, Hans-Uwe, und Nadia Kutscher. 2004. Informelle Bildung online: Perspektiven für Bildung, Jugendarbeit und Medienpädagogik. Weinheim u. München: Beltz Juventa.

Paus-Haase, Ingrid, Claudia Lampert, und Daniel Süss. 2002. «Zu Selbstverständnis der Medienpädagogik». In Medienpädagogik in der Kommunikationswissenschaft. Positionen, Perspektiven, Potenziale, hrsg. v. Ingrid Paus-Haase, Claudia Lampert, und Daniel Süss, 9-18. Wiesbaden: Westdeutscher Verlag.

Pietraß, Manuela. 2015. «Informelles Lernen und Medienpädagogik». In Handbuch Informelles Lernen, hrsg. v. Matthias Rohs, 123-142. Wiesbaden: Springer VS.

Rohs, Matthias, Ricarda Bolten, und Jonathan Kohl. 2017. «Medienpädagogische Kompetenzen in Kompetenzbeschreibungen für Erwachsenenbildner*innen». Beiträge zur Erwachsenenbildung 5/2017. https://kluedo.ub.uni-kl.de/files/4690/_Beitraege_zur_EB_5_final.pdf.

Scharnberg, Gianna, Anne-Cathrin Vonarx, Michael Kerres, und Karola Wolff. 2017. «Digitalisierung der Erwachsenenbildung in Nordrhein-Westfalen - Herausforderungen und Chancen wahrnehmen». Magazin erwachsenenbildung.at. 30/2017, 2-12.

Schelhowe, Heidi. 2008. «Digitale Medien als kulturelle Medien: Medien zum Be-Greifen wesentlicher Konzepte der Gegenwart». In Pädagogische Medientheorie, hrsg. v. Johannes Fromme, und Werner Sesink, 95-113. Wiesbaden: VS Verlag.

Schell, Fred. 1989. Aktive Medienarbeit mit Jugendlichen. München.

Schiefner-Rohs, Mandy, und Sandra Hofhues. 2017. «Prägende Kräfte. Medien und Technologie(n) an Hochschulen.» In Medien, Bildung und Wissen in der Hochschule, hrsg. v. Johannes Othmer, Andreas Weich und Katharina Zickwolf, S. 239-254. Wiesbaden: Springer.

Schludermann, Walter. 2002. «Medienmündigkeit als gesellschaftliche Herausforderung». In Medienpädagogik in der Kommunikationswissenschaft. Positionen, Perspektiven, Potenziale, hrsg. v. Ingrid Paus-Haase, Claudia Lampert, und Daniel Süss, 49-53. Wiesbaden: Westdeutscher Verlag. 
Schulmeister, Rolf. 2013. «Einleitung: Der Beginn und das Ende von OPEN. Chronologie der MOOC-Entwicklung». In MOOCS - Massive Open Online Courses: offene Bildung oder Geschäftsmodell?, hrsg. v. Rolf Schulmeister, 17-59. Münster: Waxmann. https://www.waxmann.com/fileadmin/media/zusatztexte/2960Volltext.pdf.

Seifert, Alexander. 2016. «Internetkompetenzen im Alter. Überlegungen zur Förderung von Medienkompetenzen im Alter». merz - Medien + Erziehung 60/2016, 66-71.

Stadler, Felix. 2016. Kultur der Digitalität. Berlin: Suhrkamp.

Stang, Richard. 2003. Medienpädagogik und Erwachsenenbildung. Annäherung an ein schwieriges Verhältnis. Vortrag im Rahmen des Medienpädagogischen Kolloquium an der Pädagogischen Hochschule Ludwigsburg am 11.12.2003. https://www.ph-ludwigsburg.de/fileadmin/subsites/1b-mpxx-t-01/user_files/StangVortrag.pdf.

Stange, Werner. 2007. «Was ist Partizipation? Definitionen - Systematisierungen». Baustein A 1.1. http://www.kinderpolitik.de/beteiligungsbausteine/pdf/a/Baustein_A_1_1.pdf.

Stölting, Erhard. 1999. «Informelle Machtbildung und Leitideen im institutionellen Wandel. Institutionenökonomie und Neuer Institutionalismus». In Überlegungen zur Organisationstheorie, Interdisziplinäre Organisationsund Verwaltungsforschung 2, hrsg. v. Thomas Edeling, Werner Jann, und Dieter Wagner, 111-131. Opladen: Leske \& Budrich.

Süss, Daniel, Claudia Lampert, und Christine W. Wijnen, Hrsg. 2010. Medienpädagogik. Ein Studienbuch zur Einführung. Wiesbaden: Springer VS.

Tillmann, Angela. 2008. Identitätsspielraum Internet: Lernprozesse und Selbstbildungspraktiken von Mädchen und jungen Frauen in der virtuellen Welt. Weinheim und München: Beltz Juventa.

Tully, Claus. 2004. Verändertes Lernen in modernen technisierten Welten. Wiesbaden: VS Verlag.

Tully, Claus. 2009. Multilokalität und Vernetzung. Beiträge zur technikbasierten Gestaltung jugendlicher Sozialräume. Weinheim, und München: Juventa Verlag.

Tulodziecki, Gerhard. 1998. «Entwicklung von Medienkompetenz als Erziehungs- und Bildungsaufgabe». Pädagogische Rundschau 6/1998, 693-709.

Tulodziecki, Gerhard. 2012. "Medienpädagogische Kompetenz und Standards in der Lehrerbildung». Jahrbuch Medienpädagogik 9, hrsg. v. Renate Schulz-Zander, Birgit Eickelmann, Heinz Moser, Horst Niesyto, und Petra Grell, 271-297. Wiesbaden.

Vollbrecht, Ralf, und Claudia Wegener. 2010. Handbuch Mediensozialisation. Wiesbaden: Springer VS. 